\title{
A propos de l'évolution d'Hymenolepis nana var. fraterna chez des hôtes intermédiaires inhabituels
}

\author{
Par R. CAVIER et N. LEGER
}

Face à l'agression parasitaire l'organisme se défend, mettant en œuvre un ensemble de mécanismes qui lui sont propres : l'hôte ne possède que des possibilités réactionnelles limitées qu'il doit adapter aux différents parasites susceptibles de s'attaquer à lui.

Les réactions décrites chez les insectes parasités par divers Métazoaires se rattachent toutes à un même type.

On distingue deux phénomènes :

$1^{\circ}$ Une réaction cellulaire : Il y a agglutination de cellules sanguines autour du parasite et encapsulement de celui-ci, avec formation ou non d'un syncytium, puis dégénérescence des cellules qui se transforment en une membrane anhiste.

On peut également observer des réactions de type cellulaire au niveau de divers tissus: ainsi les microfilaires peuvent s'enkyster dans les muscles des moustiques vecteurs.

$2^{\circ}$ Une mélanisation: Cette mélanisation du parasite est un phénomène qui semble être assez général chez les Insectes.

Les parasites les plus pigmentés sont souvent morts, sans que cela signifie pour autant que le pigment ne se soit déposé qu'après la mort.

Ce pigment, dont la couleur varie du jaune au noir, se développe à l'intérieur de la capsule cellulaire, toujours dans les couches les plus internes et à la surface du parasite chez lequel il n'imprègne jamais les téguments en profondeur.

Ajoutons que la possibilité de réactions de type humoral a été également avancée sans qu'on ait jamais pu en faire la preuve.

En ce qui concerne les Cestodes parasites des Insectes, la seule information que nous ayons pu recueillir nous a été fournie par Chen [1]. Travaillant sur des puces parasitées par des cysticercoïdes de Dipylidium caninum, il décrit chez celles-là un double mécanisme réactionnel :

$1^{\circ}$ un encapsulement plus ou moins total de certains cysticercoïdes par des amoebocytes de l'insecte ; 
$2^{\circ}$ la formation à l'intérieur de la capsule d'un pigment mélanique.

C'est cette absence quasi totale d'observations relatives aux Cestodes, face à l'abondance des descriptions des réactions observées avec les Nématodes et les Insectes parasites, qui nous a incités à entreprendre nos travaux.

\section{I. - MATERIEL D'ETUDE ET METHODES}

\section{A. - Le Parasite}

Notre étude a porté sur Hymenolepis nana var. fraterna. Rappelons que ce Cestode possède deux voies d'évolution possibles:

1) un cycle direct s'effectuant entièrement chez des Rongeurs qui abritent successivement la forme cysticercoïde et la forme adulte ;

2) un cycle indirect au cours duquel le cysticercoïde est hébergé par divers insectes qui servent d'hôtes vecteurs pour la transmission aux rongeurs.

\section{B. - Les Hôtes}

a) Les hôtes intermédiaires usuels sont :

- des Siphonaptères: Pulex irritans, Ctenocephalides canis, Nosopsyllus fasciatus et Xénopsylla cheopis;

- des Coléoptères : Tribolium confusum, Tenebrio molitor et Tenebrio obscurus.

Personnellement, nous avons également réussi à infester des grillons domestiques (Gryllus domesticus) par la voie buccale en utilisant la technique décrite plus loin.

b) Les hôtes expérimentaux. Schiller [3], essayant de parasiter par la voie buccale des blattes (Periplaneta americana), échoua. Reprenant ses expériences, nous avons vérifié la résistance de ces insectes à l'infestation par la voie buccale.

En revanche, en introduisant directement dans leur cavité générale des embryons éclos « in vitro », nous avons pu constater qu'Hymenolepis nana était capable de se développer chez ces insectes et de donner le cysticercoïde.

\section{C. - Les Techniques}

a) INFESTATION PAR LA VOIE BUCCALE :

Nous avons utilisé la technique décrite par Schiller [3] :

$1^{\circ}$ Obtention des aufs: On sélectionne des souris hébergeant des Cestodes sexuellement mûrs, c'est-à-dire éliminant des œufs dans leurs fèces.

Les helminthes sont recueillis dans les dernières portions du grêle, après autopsie des animaux, et on les dispose dans des boîtes de Pétri contenant quelques millilitres d'eau ordinaire. Le tout est abandonné vingt-quatre heures au réfrigérateur. 
$2^{\circ}$ Infestation des Insectes: On garnit le fond d'un petit bocal de rondelles de papier-filtre et on y verse les fragments d'Hymenolepis. On dépose ensuite dans le bocal les insectes maintenus à jeun depuis au moins quarante-huit heures. Le bocal est placé à l'étuve à $30^{\circ} \mathrm{C}$ et y est maintenu pendant toute la durée de l'infestation. Le repas infestant dure vingt-quatre heures. On retire alors les rondelles de papier-filtre et on donne de la nourriture aux animaux.

La température joue un rô'e capital dans le développement du parasite. Voge et Turner [7], travaillant sur Hymenolepis diminuta chez Tribolium confusum, ont montré que le développement des cysticercoïdes n'était possible qu'entre 15 et $37^{\circ} \mathrm{C}$. Aux températures plus basses on n'observe pas de déve'oppement; aux températures plus élevées on obtient des cysticercoïdes présentant des anomalies morphologiques et dénués de tout pouvoir infestant pour la Souris. Pour ces auteurs la température la plus favorable est de $30^{\circ} \mathrm{C}$. C'est cette température que Voge et Heyneman [5,6] adoptèrent lorsque, plus tard, ils travaillèrent sur Hymeno'epis nana chez Tenebrio molitor. Schiller [3], lors de ses expériences sur Tribolium confusum donne comme température optimum d'infestation : $28^{\circ} \mathrm{C}$. En ce qui nous concerne, infestant Tenebrio molitor, nous avons obtenu constamment un fort pourcentage d'infestation ( 80 à $100 \%$ ) en opérant à $30^{\circ} \mathrm{C}$.

\section{b) INFEstation Par La Voie intra-Cavitaire.}

Nous avons utilisé une technique inspirée de celle employée par Voge et Graiwer [4] pour infester des larves de Tenebrio molitor. Les exemplaires adultes d'Hymenolepis nana sont obtenus par autopsie de souris parasitées et ouverture des dernières portions de l'iléon. Les vers prélevés sont broyés au mortier avec quelques millilitres de liquide de Ringer. Le broyat obtenu est agité pendant cina minutes avec des billes de verre ; cette opération a pour but de briser la coque externe de l'œuf. On ajoute alors un volume égal de la solution suivante :

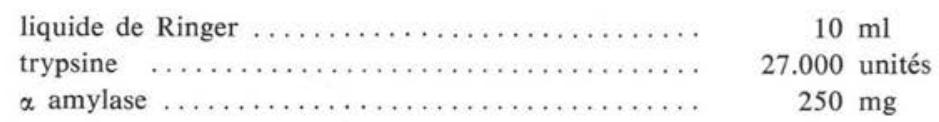

afin de provoquer la lyse de la membrane hyaline de l'œuf et la rupture de l'embryophore. Le tout est abandonné une heure à une heure et demi à l'étuve à $30^{\circ} \mathrm{C}$.

A ce moment on observe dans le milieu des embryons hexacanthes libres, parmi de nombreuses coques brisées, des œufs conservant des lambeaux plus ou moins importants de membrane hyaline, des embryons encore enfermés dans leur embryophore. La plupart de ces derniers montrent une très grande activité, alors qu'une fois libérés ils deviennent aussitôt immobiles.

Lors de nos premiers essais nous injections directement ce mélange aux blattes. Mais nous obtenions alors une mortalité de près de $100 \%$ au bout de vingt-quatre heures. Par la suite nous avons modifié notre technique afin d'éliminer les enzymes.

Nous laissons sédimenter la suspension d'embryons, et, après avoir éliminé le liquide surnageant, nous procédons à trois lavages successifs par sédimentation dans la 
solution de Ringer. Les embryons, remis en suspension dans le même liquide, sont introduits dans la cavité générale des blattes. Pour ce faire, on utilise une aiguille très fine $(5 / 10)$ montée sur une petite seringue et on pratique l'injection entre deux sternites abdominaux. De cette façon l'injection est assez bien supportée, quoiqu'on obtienne toujours une certaine mortalité (de 1 pour 10 à 1 pour 4 ) parmi les insectes traités.

Les animaux infestés sont placés à l'étuve à $30^{\circ} \mathrm{C}$, et on surveille chaque jour le développement des embryons par dissection dans du soluté physiologique.

Dans ces conditions nous avons obtenu une infestation quasiment constante, aussi bien chez les adultes que chez les larves de Periplaneta. Les cysticercoïdes sont présents en grand nombre et très actifs. Toutefois on observe un retard de développement et des anomalies morphologiques. Alors que chez le ténébrion infesté par la voie buccale le cysticercoïde acquiert son aspect définitif en quatre à cinq jours, ici, au bout de six jours, nous n'avions pas obtenu l'invagination du rostellum et les crochets n'existaient qu'à l'état d'ébauches. Une de nos blattes infestées a été disséquée au bout de huit jours. Elle contenait de nombreux cysticercoïdes. A ce stade les crochets étaient bien développés, mais le cysticercoïde conservait un aspect anormal, avec un scolex grêle n'occupant qu'une partie de la cavité de la capsule.

Les mêmes expériences ont été menées chez d'autres blattes : Leucophaea maderae, et chez ces dernières le pouvoir infestant des cysticercoïdes obtenus a été vérifié par administration à des souris chez lesquelles le parasite s'est développé jusqu'au stade adulte. L'aspect des cysticercoïdes observé chez Leucophaea était sensiblement différent de celui des exemplaires provenant de Periplaneta. En particulier, ici, le scolex n'était pas étiré et grêle mais au contraire comme écrasé contre la paroi interne de la capsule.

\section{II. - LES REACTIONS OBSERVEES}

\section{A) Chez les Hôtes infestables par la voie buccale}

Que ce soit chez Tenebrio molitor ou chez Gryllus domesticus, nous n'avons jamais pu mettre en évidence de phénomènes réactionnels. Des ténébrions et des grillons ont été infestés par la voie intracavitaire. Les cysticercoïdes s'y sont normalement développés et là non plus nous n'avons pas observé de réactions de la part de l'hôte.

\section{B) Chez les Hôtes non infestables par la voie buccale}

En revanche, chez les blattes (Periplaneta et Leucophaea) que nous avions contraintes à héberger le parasite, nous avons pu observer des formations tout à fait comparables à celles que nous évoquions au début de notre exposé.

Dès nos premières expériences sur Periplaneta americana, nous avions été frappés par l'apparition chez celles-ci de granulations noirâtres, abondantes, visibles à l'œil nu lors de la dissection, de telle sorte qu'il nous était possible de reconnaître immédiatement les animaux infestés. 

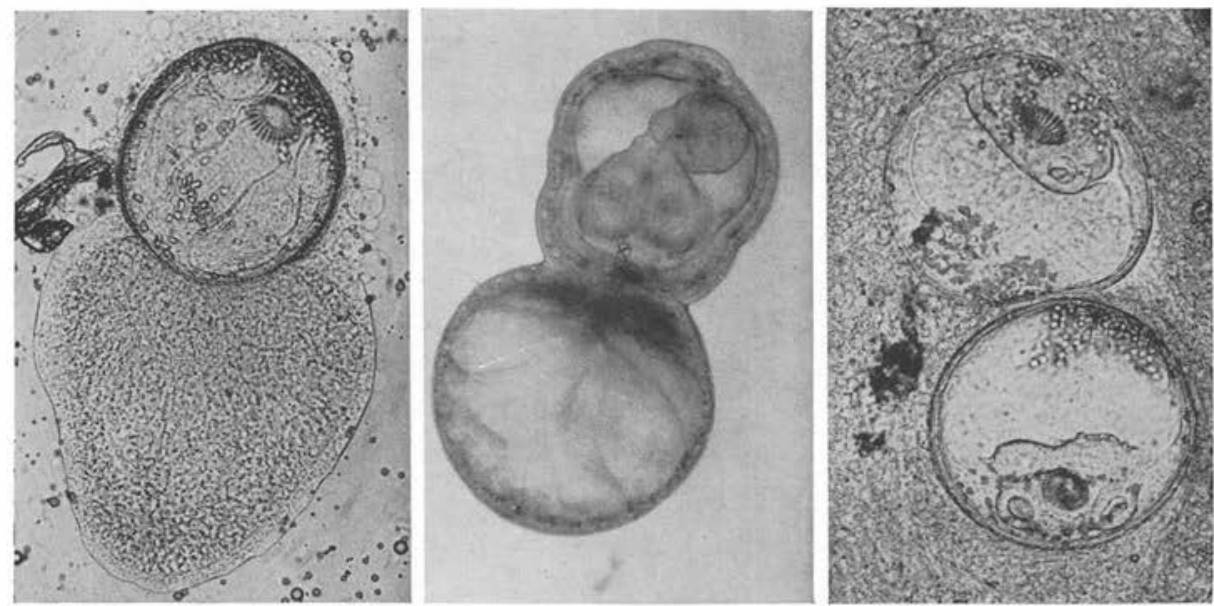

Planche 1

Pното 1. - Cysticercoïde de six jours chez Tenebrio molitor

Рното 2. - Cysticercoïde de six jours chez Périplaneta americana

Pното 3. - Cysticercoïdes de quatorze jours chez Leucophaea moderae

$\mathrm{Au}$ microscope, ces granulations se présentent comme des amas de pigment noir entourés d'une épaisse capsule non pigmentée, de nature cellulaire. Souvent on observe, au centre de ces capsules, des coques vides et des œufs entiers plus ou moins recouverts de pigment. Nous avons obtenu des résultats analogues chez Leucophaea. Alors que nous n'observions rien de particulier au bout de trois jours, au septième jour de l'infestation de nombreux cysticercoïdes étaient recouverts de dépôts noirâtres et on retrouvait les capsules cellulaires déjà décrites chez Periplaneta. Les formations les plus caractéristiques furent observées chez les animaux sacrifiés au bout de quatorze jours. Les scolex y étaient réunis par groupes d'une dizaine et plus, au sein d'une capsule commune formée de couches de cellules plus ou moins concentriques, et dont le centre était occupé par un amas de granulations noirâtres, renfermant des œufs et des débris de coques. La queue de ces cysticercoïdes avait totalement disparu. Certaines de ces formations mesuraient plusieurs millimètres. Souvent elles étaient adhérentes aux téguments et aux organes internes de l'insecte.

Signalons qu'en dépit de l'importance des réactions observées Leucophaea maderae semble bien supporter le parasitisme : au bout de quatorze jours, tous les insectes traités étaient vivants et avaient un comportement apparemment normal.

\section{III. - CONCLUSIONS}

$1^{\circ}$ Il est possible de faire évoluer des cysticercoïdes d'Hymenolepis nana chez des espèces qui ne sont pas infestables par ingestion d'œufs: il suffit d'introduire les 

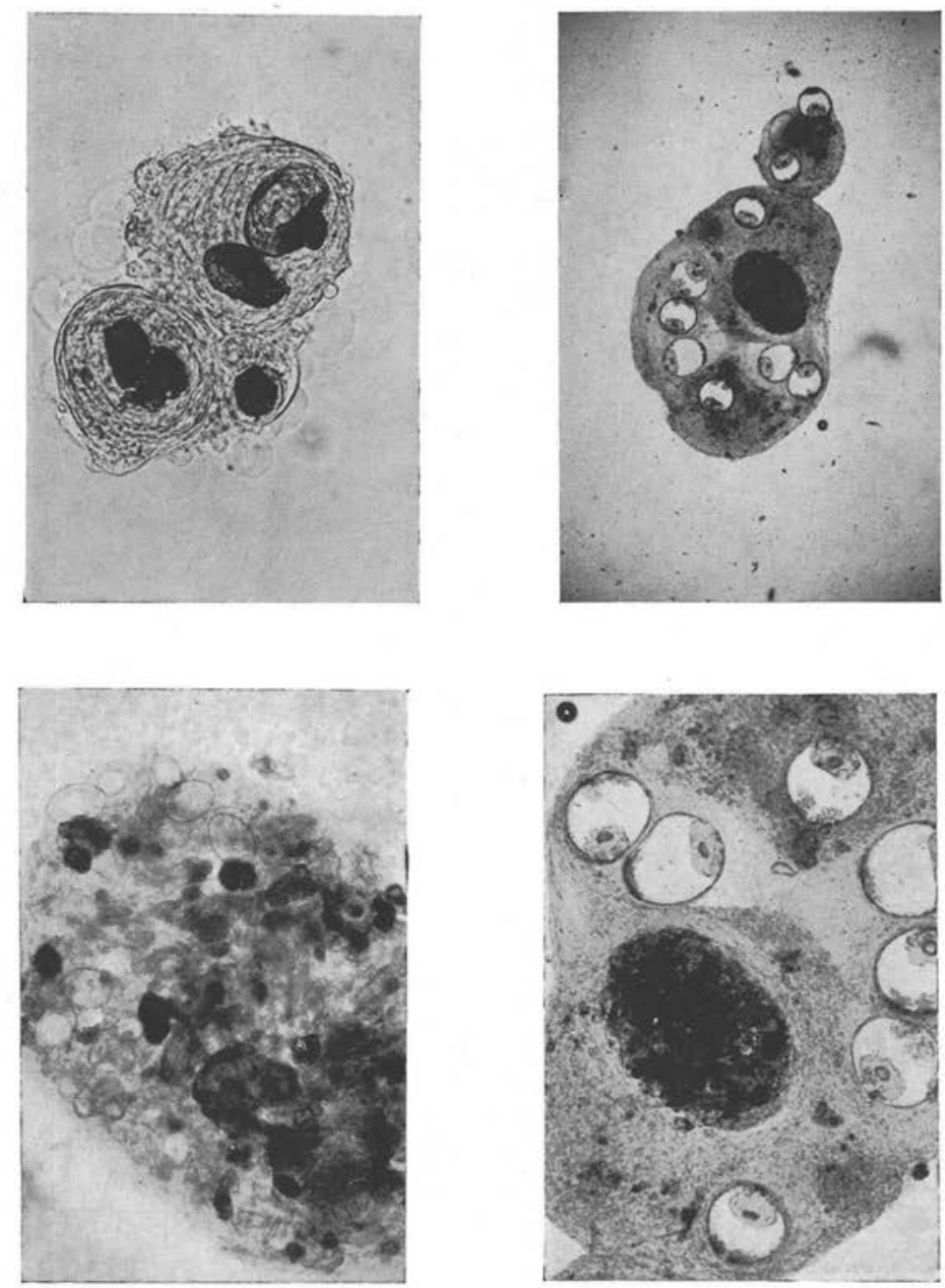

\section{Planche 2}

Рното 4. - Dépôts pigmentaires et capsules autour d'œufs d'Hymenolepis nana chez Periplaneta americana

Phoтоs 5, 6 et 7. - Formation observée chez Leucophaea ou quatorzième jour de l'infestation (aspect général et détails)

embryons éclos « in vitro» directement dans la cavité générale de ces insectes dont le milieu intérieur doit contenir les facteurs nécessaires à la survie et à la croissance du parasite. Si ceux-là ne sont pas infestables par la voie buccale, c'est donc que l'obstacle 
se situe au niveau du tube digestif, soit que celui-ci ne constitue pas un milieu favorable à la libération, à partir de l'œuf, de l'embryon hexacanthe, soit que la paroi intestinale s'oppose au passage de ce dernier.

$2^{\circ}$ Chez les hôtes normaux le parasite n'engendre aucune réaction. Au contraire, chez les hôtes anormaux, non infestables par la voie buccale, on observe un encapsulement et un dépôt de pigment autour des cysticercoïdes.

Ici se pose le problème de l'adaptation du parasite à son hôte : d'une façon générale les parasites des insectes seraient-ils trouvés encapsulés plus souvent chez les hôtes inhabituels que chez les hôtes normaux?

Dans une revue générale des réactions des insectes aux métazoaires, à propos de certains Spiruroïdés parasites, Salt [2] posait récemment cette question. Il fait également remarquer que Chen [1] effectuait une partie de ses travaux sur Ctenocephalides felis, qui, bien qu'il soit un hôte habituel de Dipyliduim caninum, n'en est pas forcément l'hôte normal. Malheureusement, bien que certaines expériences aient été parfois effectuées sur Ctenocephalides canis, hôte le plus fréquent du parasite, l'article de Chen ne distingue pas les observations faites avec les deux espèces.

Pour nous, nous pouvons nous demander si l'absence totale de réaction des hôtes normaux à Hymenolepis nana ne serait pas une preuve de la parfaite adaptation de ce parasite à ses hôtes?

\section{Bibliographie}

1. CHEN (H. T.), 1934. - Reactions of Ctenocephalides felis to Dipylidium caninum. Zeit. $f$. Parasit., 6, p. 603-637.

2. Salt (G.), 1963. - The defense reactions of Insects to metazoan parasites. Parasit., 53, p. $527-642$.

3. SCHiller (E. L.), 1959. - Experimental studies on morphological variation in the Cestode genus Hymenolepis. I. Morphology and development of the cysticercoïd of Hymenolepis nana in Tribolium confusum. Exp. Parasitol., 8, p. 91-118.

4. Voge (M.) et Graiwer (M.), 1964. - Development of onchospheres of Hymenolepis diminuta hatched in vivo and in vitro in the larvae of Tenebrio molitor. J. Parasit., 50 , p. $267-270$.

5. Voge (M.) et Heyneman (D.), 1957. - Development of Hymenolepis nana and Hymenolepis diminuta (Cestoda, Hymenolepididae) in the intermediate host Tribolium confusum. Univ. Calif. Publ. Zool., 59, p. 549-579.

6. Voge (M.) et Heyneman (D.), 1958. - Effect of high temperature on the larval development of Hymenolepis nana and Hymenolepis diminuta. J. Paras., 44, p. 249-260.

7. Voge (M.) et Turner (J. A.), 1956. - The effect of different temperatures on the development of Hymenolepis diminuta in Tribolium confusum. J. Paras. 42 ( $4^{e}$ sect. 2), p. 31 et Exp. Paras., 5, p. 580-586.

(Chaire de Parasitologie, Faculté de Pharmacie de Paris, 4, avenue de l'Observatoire Paris, $6^{\circ}$ ) 